\title{
Caracterización morfológica de 160 accesiones de ajonjolí (Sesamum indicum L.) del banco de germoplasma de
}

\section{Colombia}

Fecha de recepción: 09 de julio de 2020

Fechadeaceptación: 03 de agosto de 2020

DOI: https://doi.org/10.19053/01228420.v17.n3.2020.11618
Johanna Paola Garnica Montaña • 1 Oscar Jair Rodríguez Rodríguez • 2 Camilo Ignacio Jaramillo-Barrios - 3 Jorge Enrique Villamil Carvajal .4 Jorge Alberto Valencia Montoya . 5

\section{Resumen}

El ajonjolí es una planta importante en la producción de aceite comestible de alta calidad por su gran valor nutricional y terapéutico. La caracterización de accesiones es necesaria para la identificación de parentales con características de interés para la obtención de nuevos materiales genéticos. Se caracterizaron 160 accesiones de ajonjoli en el Centro de Investigación Nataima, municipio de El Espinal (Tolima), manejadas por el Sistema de Bancos de Germoplasma de la Nación para la Alimentación y la Agricultura, de las cuales, 136 fueron colectadas en 25 países. La caracterización incluyó 81 descriptores: 30 cuantitativos y 51 cualitativos. Se examinaron factores para datos mixtos y se generaron grupos homogéneos mediante análisis de conglomerados para cinco grupos de variables: planta y plántula, tallo y raíz, hoja, semilla y cápsula y flor. Se seleccionaron 25 variables discriminantes de las estructuras anteriores y se realizó un nuevo análisis de conglomerados que generó cinco agrupaciones principales influenciadas por las variables cualitativas: tipo y hábito de crecimiento, color del tallo, forma de la hoja, tipo de ramificación, color interno de la corola, posición de las cápsulas y color de la semilla, y cuantitativas: altura, longitud de internudos, número de ramas principales y secundarias, número de cápsulas por nudo, número de cápsulas por planta, número de semillas por cápsula, peso de 1000 semillas y rendimiento. La estructura de la agrupación no presentó influencia por la procedencia de las accesiones. Las accesiones TEHUANTEPEC SELEC, LAJ-M-67, L-AJ-M-51, PARDO PELUDO, BLANCO CRIOLLO, NP-61, BACO, INSTITUTO 104, CHINO ROJO, R 198 y ACARIGUA presentaron características de interés que diferencian los grupos formados. Las variables discriminantes y los materiales identificados permiten la generación de variedades para futuros programas de mejoramiento en Colombia.

Palabras clave: accesiones, morfología, semillas oleaginosas, germoplasma, sésamo.

\section{Morphological characterization of 160 accessions of sesame (Sesamum indicum L.) from the Colombian germplasm bank}

\section{Abstract}

Sesame seed is an important plant in the production of high-quality edible oil for its high nutritional and therapeutic value. The characterization of accessions is necessary for the identification of parents with characteristics of interest for obtaining new genetic materials. 160 accessions of sesame (Sesamum indicum L.) were characterized in the Nataima, municipality of El Espinal (Tolima), managed by the National Germplasm Banks System for Food and Agriculture, of which, 136 are from 25 countries; 81 descriptors were used, 30 quantitative and 51 qualitative. Factor analysis of mixed data was performed, and homogeneous groups were generated by cluster analysis for five groups of variables: plant and seedling, stem and root, leaf, seed and capsule and flower. 25 discriminating variables were selected from the previous structures and a new cluster analysis was performed. This generated five main groupings influenced by qualitative variables: habit, type of growth and height in plant and seedling; type of branching, pubescence and number of primary branches in stem and root; position, petiole length and leaf width; position of the capsule, color of the cover and number of capsules in seed and capsule and coloration of the corolla, extrafloral nectary and number of flowers per armpit in flower. The accessions TEHUANTEPEC SELEC, L-AJ-M-67, L-AJ-M-51, BROWN HAIR, CREOLE WHITE, NP-61, BACCHUS, INSTITUTE 104, RED CHINESE, R 198 and ACARIGUA present characteristics of interest that differentiate the formed groups. The discriminant variables and the identified materials allow the generation of varieties for future breeding programs in Colombia.

Keywords: accessions, morphology, oilseeds, germplasm, sesame.

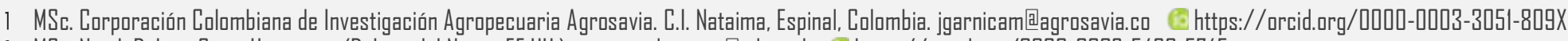

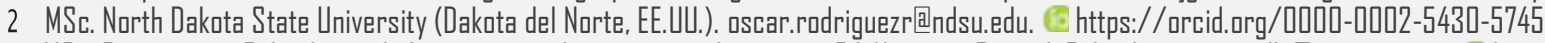

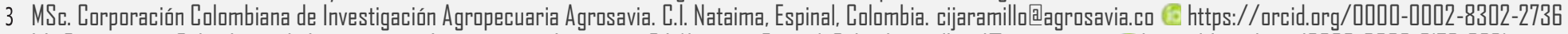

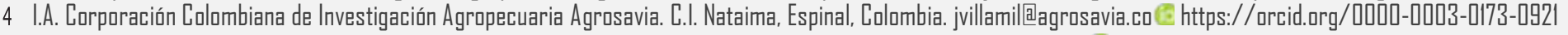

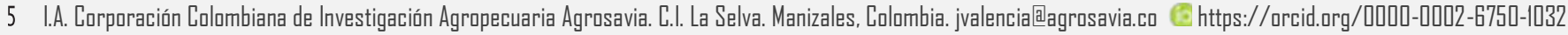




\section{Introducción}

El ajonjolí o sésamo (Sesamum indicum L.) es una especie oleaginosa que pertenece a la familia Pedaliácea. Etiopía es considerado su centro de origen, pero cuenta con distintos centros de diversificación en el continente asiático. Es importante en la producción de aceite comestible de alta calidad por su gran valor nutricional y terapéutico (Hata et al., 2012), se adapta a climas tropicales y subtropicales generando altos rendimientos. Es autógama con poblaciones generalmente compuestas de individuos homocigotos, por tanto, el avance genético se obtiene con la selección de individuos después de varias generaciones y se reproduce por autopolinización (Bedigian et al., 1986; Morris, 2009; Furat \& Uzun, 2010).

Se han realizado estudios que caracterizan la diversidad morfológica del germoplasma de ajonjolí e indican una amplia diversidad en caracteres relacionados con altura, ramificación, cápsula y semillas (Bedigian et al., 1986; IPGRI \& NBPGR, 2004; Pham et al., 2010). Las colecciones más importantes de germoplasma en el mundo se conservan en la India, China, Rusia y Estados Unidos (Mazzani et al., 2007). Sin embargo, el éxito de los programas depende de la diversidad del recurso genético.

En Colombia, el Sistema de Bancos de Germoplasma de la Nación para la Alimentación y la Agricultura (SBGNAA) conserva semilla de 160 accesiones de ajonjolí a $0{ }^{\circ} \mathrm{C}$ en el Centro de Investigación Tibaitatá de Agrosavia (Valencia et al., 2010). La primera evaluación morfológica la realizó Rey (1999) en el año 1994; él evaluó el banco de germoplasma de ajonjolí con 11 características morfoagronómicas cuantitativas en 130 accesiones compuestas por colecciones regionales, introducciones y variedades colombianas; además, logró generar 10 variedades mejoradas (Ica Pacandé, Ica Ambalá, Ica Matoso, Sesica M-11, Chino Rojo, Pepino, Habano, Pico de perdiz, Aceitera y Pardo peluda); posteriormente, Pérez-Bolaños y Salcedo-Mendoza (2018) identificaron los componentes de rendimiento en el departamento de Sucre para los cultivares Criollo, ICA Matoso y Chino Rojo.

La producción de ajonjolí en el país ha tenido periodos fluctuantes. En la década de los 60 se sembraron alrededor de 80000 ha y en los años 70 se estabilizó en 40000 ha, siendo los departamentos del Tolima, Cesar y Magdalena los principales productores; en 1980 disminuyó a 29300 ha año-1 y en los 90 se produjeron 15000 ha año-1 (Rey, 1999). Durante el 2008 se redujo la producción a 2720 ha año-1, de las cuales el $84,7 \%$ se obtuvo en el Caribe colombiano y el MINTIC (2018) reportó 1670 ha con una producción de 1386 t y un rendimiento de 5,01 tha-1.

El presente trabajo tuvo como objetivo caracterizar las accesiones de ajonjolí conservadas en el Banco de Germoplasma de Colombia para clasificar la variabilidad genética presente sobre la base de caracteres cualitativos y cuantitativos mediante un análisis factorial de datos mixtos (AFDM) y análisis de conglomerados (AC) para diferenciar clases homogéneas en cinco grupos de variables: planta y plántula, tallo y raíz, hoja, semilla y cápsula y flor. Asimismo, realizar un AC conjunto entre caracteres morfológicos y componentes de rendimiento. Finalmente, identificar accesiones promisorias como fuentes genéticas para programas de mejoramiento. Este estudio se convierte en la caracterización morfológica más completa del banco de germoplasma de ajonjolí en Colombia. 


\section{Metodología}

El estudio se desarrolló durante el 2012 en las instalaciones del Centro de Investigación Nataima de la Corporación Colombiana de Investigación Agropecuaria (Agrosavia), situado a 04¹' $58,2^{\prime \prime}$ latitud norte y $74^{\circ} 57^{\prime} 97,3^{\prime \prime}$ longitud oeste, ecorregión de Valles Interandinos, municipio de El Espinal (Tolima) con una precipitación media anual de $1350 \mathrm{~mm}$, temperatura media de $28^{\circ} \mathrm{C}$, suelos franco-arenosos y formación ecológica de bosque seco tropical (bsT).

Se establecieron parcelas de evaluación con un área de $18 \mathrm{~m}^{2}$ para cada accesión, cada una conformada por cuatro surcos de $5 \mathrm{~m}$, con una distancia de $0,8 \mathrm{~m}$ entre surcos y $0,25 \mathrm{~m}$ entre plantas. En los surcos se prepararon 20 sitios con dos semillas cada uno y la siembra se hizo a $3 \mathrm{~cm}$ de profundidad para un total de 160 semillas. Se realizó un raleo a los 30 días después de la siembra, asegurando una población de 50000 plantas ha-1 y se registró un porcentaje promedio de germinación para todas las accesiones de 89,12\%. El manejo agronómico incluyó labores de riego, manejo de plagas y enfermedades, fertilización, control de arvenses y manejo poscosecha de manera homogénea.

Se caracterizaron en campo 160 accesiones (Tabla 1) manejadas por el Sistema de Bancos de Germoplasma de la Nación para la Alimentación y la Agricultura (SBGNAA), clasificadas en las categorías colecciones nacionales, introducciones o variedades, de las cuales, 136 fueron colectadas en 25 países y 24 no tienen información concreta sobre el origen de estos materiales (Tabla 2).

\section{A. Variables morfológicas}

La evaluación de los caracteres morfológicos se llevó a cabo con base en los descriptores para sésamo propuestos por el IPGRI y NBPGR (2004). Se caracterizaron cinco plantas por accesión de los surcos centrales evitando el efecto borde. La evaluación se inició desde la emergencia de cotiledones hasta el secado de semillas con 81 variables morfológicas, agronómicas y de rendimiento (51 cualitativas y 30 cuantitativas) organizadas de acuerdo con la formación de la planta y plántula, y las estructuras (tallo y raíz, hoja, flor, cápsula y semilla) (Figura 1). La variable rendimiento de semilla $\left(\mathrm{kg} \mathrm{ha}^{-1}\right)$ se determinó a partir del peso total de semillas en cinco plantas centrales por parcela, las cuales se extrapolaron a una densidad de 50000 plantas por hectárea.

Del total de variables, se eliminaron siete caracteres, de los cuales, cuatro son monomórficos, tres con presencia (foveola en la flor, pubescencia en brácteas y color del filamento de las anteras) y uno con ausencia (glándula de la hoja), dos variables presentaron $\geq 30 \%$ en datos faltantes (color y dehiscencia de la cápsula seca) y la variable color de cubierta de la semilla es $100 \%$ dependiente. El análisis final se efectuó con un total de 74 caracteres morfológicos (Tabla 3). 
Tabla 1. Listado de accesiones de ajonjolí del Banco de Germoplasma de Colombia

\begin{tabular}{|c|c|c|c|c|c|}
\hline N.으 & Entrada & NN.음 & Entrada & NN.음 & Entrada \\
\hline 1 & ICA MATOSO & 55 & PI-278161 & 109 & TEHUANTEPEC \\
\hline 2 & SESICA M-11 & 56 & PI-278160 & 110 & VENEZUELA 51 \\
\hline 3 & ICA AMBALA & 57 & PI-256522 & 111 & VENEZUELA 52 \\
\hline 4 & ICA PACANDE & 58 & PI-256521 & 112 & PALOMA \\
\hline 5 & CHINO ROJO & 59 & PI-251704 & 113 & L-609 \\
\hline 6 & CRIOLLO NEGRO & 60 & PI-251703 & 114 & BACO \\
\hline 7 & INTRODUCCIÓN CHINA & 61 & PI-250099 & 115 & S-17 \\
\hline 8 & R 198 & 62 & PI-238992 & 116 & $X-102$ \\
\hline 9 & BLANCO CRIOLLO & 63 & PI-234428 & 117 & VAR.RADIATUM \\
\hline 10 & VERDE NACIONAL & 64 & PI-234427 & 118 & NP-61 \\
\hline 11 & CALIMA 2018 & 65 & PI-251034 & 119 & NP-62 \\
\hline 12 & CALIMA 2015 & 66 & PI-232033 & 120 & C-1036 \\
\hline 13 & CALIMA 2024 & 67 & $\mathrm{PI}-211088$ & 121 & ES-17 \\
\hline 14 & PI-304259 & 68 & PI-207665 & 122 & ES-117 \\
\hline 15 & PI-200427 & 69 & PI-207664 & 123 & ES-2 \\
\hline 16 & INSTITUTO 7 & 70 & PI-200428 & 124 & ES-84 \\
\hline 17 & INSTITUTO 8 & 71 & PI-200114 & 125 & TIL No1 \\
\hline 18 & INSTITUTO 9 & 72 & PI-200113 & 126 & MURG-1 \\
\hline 19 & INSTITUTO 9-T1-2 & 73 & Pl-188815 & 127 & JUBILEJNESS \\
\hline 20 & INSTITUTO 25 & 74 & PI-186511 & 128 & UNYYMK-81 \\
\hline 21 & LERDO & 75 & PI-186510 & 129 & $\begin{array}{l}\text { TEHUANTEPEC III } \\
\text { SELEC }\end{array}$ \\
\hline 22 & L-AJ-M-43 & 76 & PI-170780 & 130 & TERAS-77 \\
\hline 23 & L-AJ-M-44 & 77 & $\mathrm{PI}-170728$ & 131 & PACHEQUE?0 \\
\hline 24 & L-AJ-M-40 & 78 & 64056 & 132 & ANNA \\
\hline 25 & L-AJ-M-39 & 79 & 64021 & 133 & EVA N ${ }^{\circ} 71$ \\
\hline 26 & L-AJ-M-39 & 80 & 82254 & 134 & LUCIDE 49 \\
\hline 27 & L-AJ-M-66 & 81 & 74010 & 135 & MEXICALI -I-LV-186 \\
\hline 28 & L-AJ-M-65 & 82 & ACARIGUA & 136 & REGIONAL CANASTO \\
\hline 29 & L-AJ-M-68 & 83 & ACEITERA & 137 & YORI-77 \\
\hline 30 & L-AJ-M-67 & 84 & ARAPATOL-56 & 138 & SUDAN \\
\hline 31 & L-AJ-M-51 & 85 & BLANCO & 139 & NATURAL MEXICO \\
\hline 32 & L-AJ-M-64 & 86 & BLEDONIA T-1 & 140 & PI 265517 \\
\hline 33 & L-AJ-M-55-1 & 87 & BLEDONIA T-2 & 141 & PI 265516 \\
\hline 34 & L-AJ-M-50-4 & 88 & CIANO 16 & 142 & CHINO HABANO \\
\hline 35 & L-AJ-M-50-1 & 89 & CIANO 27 & 143 & LIGERITO ATLANTICO \\
\hline 36 & L-AJ-M-3-1 & 90 & CIANO 95 & 144 & LIGERITO CORDOBA \\
\hline 37 & R 193 & 91 & COAHUILOTE & 145 & L-507 \\
\hline 38 & L-AJ-M-66-4 & 92 & DULCE & 146 & MAPOARAL \\
\hline 39 & L-AJ-M-64-8 & 93 & EARIN RUSSIAN & 147 & NEGRO \\
\hline 40 & YUCATAN (México) & 94 & GENESA & 148 & NEGRO POLVORA \\
\hline 41 & VENEZUELA-1 & 95 & GLAUCA & 149 & ORO \\
\hline 42 & VENEZUELA 2 & 96 & GUACARA & 150 & PEPINO \\
\hline 43 & PI-157158 & 97 & HABANO & 151 & PICO PERDIZ \\
\hline 44 & PI-153509 & 98 & HINAMAR & 152 & $\begin{array}{l}\text { REGIONAL } \\
\text { GUERRERO }\end{array}$ \\
\hline 45 & PI-153510 & 99 & INSTITUTO 5 & 153 & REGIONAL SAYATE \\
\hline 46 & PI-154308 & 100 & INSTITUTO 15 & 154 & AMBALA \\
\hline 47 & $\mathrm{Pl}-156618$ & 101 & INSTITUTO 15 T1-2 & 155 & AMBALA \\
\hline 48 & Pl-157156 & 102 & INSTITUTO 15 T1-3 & 156 & ES-7 \\
\hline 49 & PI-164901 & 103 & INSTITUTO 101 & 157 & ES-719 \\
\hline 50 & PI-16385 & 104 & INSTITUTO 103 & 158 & PARDO PELUDO \\
\hline 51 & PI-158029 & 105 & INSTITUTO 104 & 159 & C-246 \\
\hline 52 & PI-311113 & 106 & INSTITUTO 106 & 160 & PI-154310 \\
\hline 53 & PI-306697 & 107 & $\mathrm{~T}-13$ & & \\
\hline 54 & PI-306696 & 108 & TARSO 8 & & \\
\hline
\end{tabular}


Tabla 2. Procedencia de accesiones de ajonjolí del Banco de Germoplasma de Colombia

\begin{tabular}{cccccc}
\hline País & Total & País & Total & País & Total \\
\hline Afganistán & 2 & Guatemala & 3 & Nigeria & 2 \\
Angola & 2 & India & 2 & Pakistán & 4 \\
Australia & 2 & Islas Vírgenes & 1 & Rusia & 1 \\
China & 2 & Israel & 2 & Taiwán & 2 \\
Colombia & 10 & Italia & 2 & Turquía & 2 \\
Corea & 1 & Japón & 2 & Unión Soviética & 2 \\
Egipto & 1 & México & 39 & Venezuela & 48 \\
Filipinas & 1 & Mozambique & 1 & Sin información & 24 \\
Grecia & 1 & Namibia & 1 & & \\
\hline
\end{tabular}
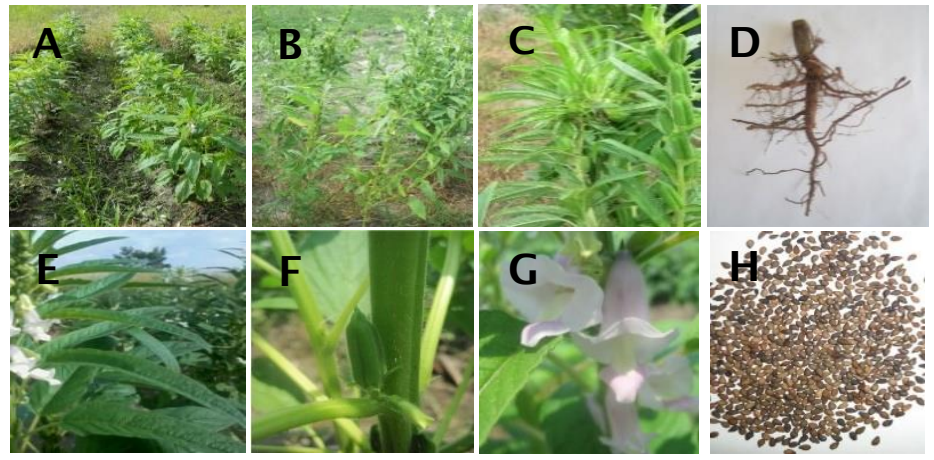

Figura 1. Estructuras de Sesamum indicum L. A. Plántula. B. Planta. C. Tallo. D. Raíz. E. Hoja. F. Cápsula. G. Flor. H. Semilla

Tabla 3. Variables de ajonjolí utilizadas en la caracterización de accesiones

\begin{tabular}{|c|c|c|c|}
\hline Grupo & Tipo & Total & Variables \\
\hline \multirow{2}{*}{$\begin{array}{l}\text { Planta y } \\
\text { plántula }\end{array}$} & Cualitativa & 6 & Color, forma, inserción y pilosidad de cotiledón; tipo y hábito de crecimiento. \\
\hline & Cuantitativa & 3 & Longitud de cotiledón y del hipocótilo, altura de la planta. \\
\hline \multirow[t]{2}{*}{ Tallo y raíz } & Cualitativa & 9 & $\begin{array}{l}\text { Forma del sistema radicular; color, pubescencia, fasciación, ramificación y rigidez del } \\
\text { tallo; patrón de ramificación, forma de la pubescencia, forma de tallo secundario } \\
\text { transversal. }\end{array}$ \\
\hline & Cuantitativa & 3 & Longitud promedio de los internudos, número de ramas principales y secundarias. \\
\hline \multirow[t]{2}{*}{ Hoja } & Cualitativa & 12 & $\begin{array}{l}\text { Color, forma, pubescencia, posición y glándula de la hoja; forma de la pubescencia; } \\
\text { perfil, margen e incisión del lóbulo de la hoja basal; color, presencia y forma de la } \\
\text { pubescencia del pecíolo; ángulo de las hojas al tallo principal. }\end{array}$ \\
\hline & Cuantitativa & 9 & $\begin{array}{l}\text { Ancho y longitud de la hoja basal, media y superior; longitud del pecíolo de la hoja basal, } \\
\text { media y apical. }\end{array}$ \\
\hline \multirow[t]{2}{*}{ Flor } & Cualitativa & 10 & $\begin{array}{l}\text { Color del labio inferior de la flor; color y desarrollo del nectario extrafloral; color completo } \\
\text { y en la punta, pubescencia del cáliz, forma de la pubescencia del cáliz; color y } \\
\text { pigmentación del interior y exterior de la corola; ausencia o presencia de la foveola, } \\
\text { pubescencia de brácteas, color del filamento de la antera y longitud del estilo. }\end{array}$ \\
\hline & Cuantitativa & 5 & $\begin{array}{l}\text { Número de flores por axila foliar, longitud de la corola, número de nudos a la primera flor, } \\
\text { longitud del lóbulo de cáliz, longitud del labio más largo. }\end{array}$ \\
\hline \multirow[t]{2}{*}{$\begin{array}{l}\text { Cápsula y } \\
\text { semilla }\end{array}$} & Cualitativa & 7 & $\begin{array}{l}\text { Color, pubescencia y tipo de pico de cápsulas por planta; dehiscencia de la cápsula en } \\
\text { maduración; textura y color de la cubierta de la semilla; forma de la semilla. }\end{array}$ \\
\hline & Cuantitativa & 11 & $\begin{array}{l}\text { Número de cápsulas por nudo, cápsulas por planta, lóculos, carpelos y semillas por } \\
\text { cápsula; longitud, ancho y grosor de las cápsulas, peso de } 1000 \text { semillas, rendimiento de } \\
\text { semilla }\left(\mathrm{kg} \mathrm{ha}^{-1}\right) \text {. }\end{array}$ \\
\hline
\end{tabular}




\section{B. Análisis estadístico}

Se realizó un análisis exploratorio inicial de todas las variables y accesiones. Los materiales PI157158, L-AJ-M-66-4, L-AJ-M-55-1, L-AJ-M-68 y ANNA presentaron un porcentaje superior al $30 \%$ en datos faltantes; por tanto, no se consideraron en el presente estudio. Las accesiones analizadas corresponden al 96,8\% de la colección.

Para el análisis estadístico, el grupo de variables se organizó de acuerdo con las estructuras y formación de la planta de ajonjolí en planta y plántula, tallo y raíz, semilla y cápsula, hoja y flor. En cada grupo se hizo un análisis factorial de datos mixtos (AFDM), el cual conjuga las técnicas de análisis de componentes principales (ACP) y análisis de correspondencias múltiples (ACM), ampliamente usadas en estudios de variabilidad genética (Hidalgo, 2003). Para identificar grupos de cultivares afines, se utilizó análisis de conglomerados (AC), con la distancia Euclidiana y el método de Ward (Mooi \& Sarstedt, 2011). Las accesiones más representativas dentro de cada clase se obtuvieron mediante la distancia entre cada accesión y el centro de su grupo (Husson et al., 2010).

Con el fin de relacionar los caracteres morfológicos con componentes de rendimiento, se seleccionaron las variables discriminantes de acuerdo con las estructuras y formación de la planta, utilizando el coeficiente de determinación $\left(\mathrm{R}^{2}\right)$ arrojado para las variables cuantitativas y el $p$-valor de la prueba chi-cuadrado de Pearson para las cualitativas del AC anterior. Un total de 25 variables fueron seleccionadas (Tabla 4). Posteriormente, se realizó un análisis de conglomerados con la distancia de Gower y el método de Ward, utilizando la función myplclust para identificar el color por origen. Los análisis se hicieron con el software $\mathrm{R}$ versión 3.4.2 (R Core Team, 2017), empleando los paquetes FactoMine $R$ en el cálculo de los resultados del AFDM, factoextra, cluster y rafalib para el análisis y visualización de los gráficos del AC.

Tabla 4. Variables discriminantes seleccionadas para AC conjunto entre caracteres morfológicos y componentes de rendimiento

\begin{tabular}{|c|c|c|c|}
\hline Grupo & Tipo & Total & Variables \\
\hline \multirow{2}{*}{$\begin{array}{l}\text { Planta y } \\
\text { plántula }\end{array}$} & Cualitativa & 2 & Tipo de crecimiento, hábito de crecimiento. \\
\hline & Cuantitativa & 1 & Altura de la planta $(\mathrm{cm})$. \\
\hline \multirow{2}{*}{$\begin{array}{l}\text { Tallo y } \\
\text { raíz }\end{array}$} & Cualitativa & 3 & Color de tallo, pubescencia de tallo, ramificación del tallo. \\
\hline & Cuantitativa & 3 & $\begin{array}{l}\text { Número de ramas principales, número de ramas secundarias, } \\
\text { longitud promedio de los internudos ( } \mathrm{mm}) \text {. }\end{array}$ \\
\hline \multirow[t]{2}{*}{ Hoja } & Cualitativa & 2 & Forma de la hoja, posición de la hoja. \\
\hline & Cuantitativa & 4 & $\begin{array}{l}\text { Ancho de la hoja basal }(\mathrm{cm}) \text {, ancho de la hoja superior }(\mathrm{cm}) \text {, } \\
\text { ancho de la hoja media }(\mathrm{cm}) \text {, longitud del pecíolo en la hoja } \\
\text { media }(\mathrm{cm}) \text {. }\end{array}$ \\
\hline Flor & Cualitativa & 3 & $\begin{array}{l}\text { Color del nectario extrafloral, desarrollo del nectario extrafloral, } \\
\text { color del Interior de la corola. }\end{array}$ \\
\hline \multirow[t]{2}{*}{$\begin{array}{l}\text { Cápsula } \\
\text { y semilla }\end{array}$} & Cualitativa & 2 & $\begin{array}{l}\text { Color de la cubierta en la semilla observada, posición de las } \\
\text { cápsulas. }\end{array}$ \\
\hline & Cuantitativa & 5 & $\begin{array}{l}\text { Número de cápsulas por nudo, cápsulas por planta, semillas por } \\
\text { cápsula, peso de } 1000 \text { semillas, rendimiento de semilla (kgha'). }\end{array}$ \\
\hline
\end{tabular}




\section{Resultados y Discusión}

El AFDM evidenció que para planta y plántula los siete primeros ejes factoriales aportaron el $64 \%$ utilizando nueve variables; tallo y raíz utilizó 11 variables, donde los diez primeros ejes factoriales contribuyeron con el 52,49\%; hoja utilizó 21 variables, los 13 primeros ejes factoriales aportaron el 46,08\%; flor empleó 15 variables, los 12 primeros ejes factoriales aportaron el 45,14\%; finalmente, cápsula y semilla utilizó 19 variables, los 12 primeros ejes factoriales contribuyeron con el $52.36 \%$. La variabilidad sintetizada para todos los grupos en los primeros ejes es baja comparada con un ACP o un AFM (Pagès, 2004) con pocas modalidades, lo cual impidió usar la matriz de coordenadas directamente para seleccionar las variables (Pacheco et al., 2009). Sin embargo, se generaron los conglomerados usando un número de factores que conservaran un alto porcentaje de variabilidad, permitiendo seleccionar las variables y modalidades discriminantes por su presencia e identidad con las clases 0 por ser excluyentes con las mismas.

\section{A. Análisis de conglomerados para cada grupo de variables}

1. Plántula y planta

El AC diferenció cinco grupos (Figura 2A). El grupo uno reunió siete accesiones $(4,52 \%)$, caracterizadas por la inserción sésil del cotiledón con coloración verde medianamente blanca, forma del cotiledón en copa plana y longitud corta del hipocótilo y crecimiento determinado. El grupo dos con 12 accesiones (7,74\%) presentó el cotiledón en forma de copa y plana, inserción sésil y pedicelada, ausencia de pilosidad y crecimiento determinado. El grupo tres incluyó 97 accesiones $(84,35 \%)$ de baja altura $(127 \pm 2,35 \mathrm{~cm})$, hábito erguido, cotiledón con pilosidad, forma plana e inserción pedicelada y tipo de crecimiento determinado. El grupo cuatro juntó 32 accesiones $(20,65 \%)$ con tipo de crecimiento indeterminado, hábito semierecto, ausencia de pilosidad en el cotiledón, porte alto $(1,53 \pm 0,528 \mathrm{~cm})$, hipocótilo largo $(64,35 \pm 2,32 \mathrm{~mm})$ y cotiledones cortos $(13,86 \pm 0,28 \mathrm{~mm})$. El grupo cinco tiene siete accesiones $(4,52 \%)$ que se distinguieron por tener hábito de crecimiento semierecto y erguido, tipo indeterminado y determinado, y longitud del cotiledón largo (16,58 $\pm 0,68 \mathrm{~mm})$.

\section{Tallo y raíz}

El AC diferenció tres grupos (Figura 2B). El grupo uno aglutinó 78 accesiones $(50,32 \%)$ con ramificación alterna en el tallo, patrón de ramificación apical, forma de pubescencia media y lisa, forma del tallo redonda en el corte transversal con pubescencia débil, rígido, color amarillo, pocas ramas primarias $(1,59 \pm 0,18)$ y secundarias $(2,70 \pm$ $0,30)$, y longitud pequeña entre los internudos $(38,94 \pm 1,38 \mathrm{~mm})$. El grupo dos incluyó 64 accesiones $(41,29 \%)$ con tallo ramificado de forma opuesta y basal, color púrpura verdoso, rigidez media a débil, forma del tallo transversal cuadrado, pubescencia corta y lisa, y longitud amplia en los internudos $(91,95 \pm 3,35 \mathrm{~mm})$. El grupo tres presentó 13 accesiones (8.38 \%) con tallo de ramificación decusado, color verde con pubescencia débil y media de forma lisa media y larga inclinada, con un alto número de ramas primarias $(14,65 \pm 1,51)$ y secundarias $(15,80 \pm 3,43)$.

\section{Hoja}

Se formaron tres grupos (Figura 2 C). El primero con 118 accesiones $(76,12 \%)$ que presentaron hojas de posición alterna con margen serrada-dentada, y en la zona basal perfil plano-cóncavo, margen entera-serrada, incisión débilausente y pubescencia débil en el pecíolo con forma corta y lisa y color amarillo, verde amarillo y verde purpura. El grupo dos incluyó 10 accesiones (6,45 \%) con hojas en posición decusada, forma ovada y cordada, margen basal 
entera, forma de pubescencia mediana-lisa y larga-inclinada, color del pecíolo amarillo con pubescencia medianafuerte, la hoja basal tiene perfil plano y no presenta incisión. El ancho de la hoja superior es de 2,92 $\pm 0,24 \mathrm{~cm}$ y la longitud del pecíolo de la hoja apical tiene 2,50 $\pm 0,14 \mathrm{~cm}$. El grupo tres reunió 27 accesiones $(17,41 \%)$ con hojas en posición opuesta, margen serrada y forma elíptica, la hoja basal mostró incisión fuerte y media en el lóbulo, perfil plano y ángulo agudo, pecíolo con pubescencia corto o medio liso, o largo e inclinado y color amarillo y verde amarillo.

\section{Flor}

Se diferenciaron 3 grupos (Figura 2D). El primero aglutinó 120 accesiones $(77,41 \%)$ caracterizadas por tener en el cáliz pubescencia media y lisa, la corola con color exterior blanco-rosa oscuro e interior blanco con manchas rosadas oscuras y foveola pigmentada a lo largo, forma de la pubescencia larga-doblada y desarrollo del nectario extrafloral rudimentario, pequeño o mediano. El grupo dos integró 20 accesiones $(12,9 \%)$ con pubescencia del cáliz corta, mediana, lisa, larga y doblada, corola pigmentada violeta oscuro o blanco rosa en el interior y con foveola pigmentada por debajo, nectario extrafloral presenta todas las formas (rudimentario, pequeño, mediano o largo), color amarillo y verde amarillo purpura, pigmentación ausente sobre la foveola, pubescencia del cáliz ausente-débil de forma corta-lisa y mediana-lisa.

El grupo tres concentró 15 accesiones $(9,67 \%)$ con color de corola en el interior blanco medianamente rosado y blanco rosa profundo y en el exterior color blanco rosa claro y blanco rosa oscuro con pigmentos a lo largo y en la foveola. El cáliz presenta en la punta color verde púrpura, pubescencia débil y mediana, diversas formas (corta-lisa, mediana-larga o larga-doblada), longitud del estilo largo y desarrollo del nectario extrafloral rudimentario y pequeño.

\section{Cápsula y semilla}

El análisis de clúster diferenció 3 grupos (Figura 2E). El primero con 25 accesiones (16,12\%), caracterizadas por ser monocapsulares, color de la cubierta de semilla café fuerte, café claro amarillenta y café rojizo oscuro y sin pubescencia en la cápsula. El grupo dos incluyó 127 accesiones $(81,93 \%)$, que presentaron cápsulas en posición múltiple (multicapsulares) con pubescencia corta, color de la cubierta de la semilla café amarillenta y textura reticularmente áspera. El grupo tres aglomeró tres accesiones $(1,93 \%)$ con características monocapsulares y multicapsulares, con cápsula bicarpelar ancha-oblonga, pubescencia corta y larga, y semilla de forma alargada con textura lisa en la cubierta.

\section{A. Análisis de conglomerados conjunto con variables discriminantes}

En la Figura 3 se presenta el agrupamiento de 155 accesiones de ajonjolí (12 variables cualitativas y 13 cuantitativas), cada conglomerado es descrito para caracterizar los aspectos relevantes. El análisis clúster diferenció cinco grupos, el primero concentró el $32,9 \%$, el segundo el 10,32\%, el tercero el $23,87 \%$, el cuarto el $7,09 \%$ y el quinto el $25,8 \%$ del total de las accesiones a un punto de corte de 1,5 . 


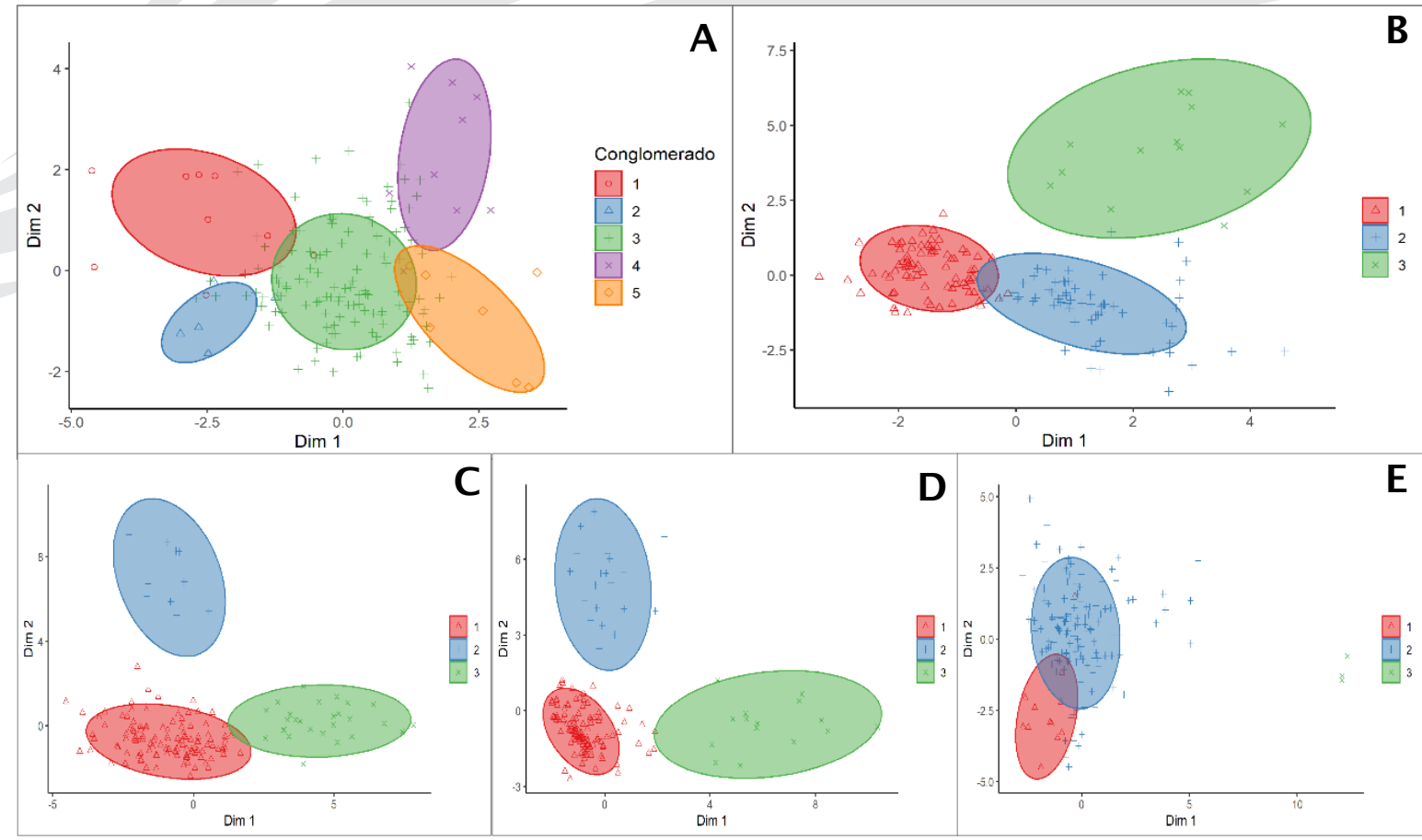

Figura 2. Agrupamiento de accesiones de ajonjolí en relación con las características de estructura y formación. A. Planta y plántula. B. Tallo y raíz. C. Hoja. D. Flor. E. Cápsula y semilla. El eje horizontal indica las coordenadas de la Dimensión 1 (Dim 1) y el eje vertical muestra las coordenadas de la Dimensión 2 (Dim 2). Cada color y cada símbolo indica el conglomerado formado. Las elipses de confianza para todas las estructuras y formación de la planta se determinaron al $95 \%$, excepto para planta y plántula al $70 \%$ y semilla y cápsula al $85 \%$.

La procedencia de las accesiones se representa en el dendrograma mediante diferentes colores: el grupo uno concentra 27 accesiones de Venezuela, 19 de México y 1 de China, Colombia, Grecia, Namibia y sin información; el grupo dos incluye 4 accesiones procedentes de Venezuela, 3 sin información, 2 de Angola y Taiwán, y 1 de Afganistán, Israel, Italia, México y Turquía; el grupo tres se conforma por 10 accesiones de Venezuela y sin información, 6 de México, 4 de Colombia, 2 de la Unión Soviética y 1 de Afganistán, Egipto, Guatemala, Israel y 1 Línea producida en el Cl Nataima; el grupo cuatro evidencia 4 accesiones de Colombia, 3 sin información y 1 de Guatemala, Islas Vírgenes, México y Venezuela; y el grupo cinco agrupa 11 accesiones de México, 6 de Venezuela, 4 de Pakistán, 2 de Australia, Guatemala, Japón, Nigeria y sin información, y 1 de China, Colombia, Corea, Filipinas, India, Italia, Mozambique, Rusia y Turquía. Lo anterior muestra que la agrupación no presenta influencia de su origen. De acuerdo con la agrupación de las accesiones se describe la siguiente conformación:

Grupo 1: Accesiones de ajonjolí de porte pequeño con crecimiento determinado, erguidas y multicapsulares

Conformado por 51 accesiones, se caracterizan por tener crecimiento determinado, y hábito erguido, el color de tallo generalmente amarillo o verde con pubescencia débil, tipo de ramificación alterna, con hojas posicionadas de forma alterna, color de nectario extrafloral externo amarillo con tonalidades claras, verdes o púrpuras, desarrollo del nectario rudimentario o pequeño, color interno de la corola blanco con manchas rosadas raramente violeta oscuro, son multicapsulares y el color de la semilla se observa desde amarillo, café hasta negro. Presentan los menores promedios de altura con $124,58 \mathrm{~cm}$, longitud internudos de $40,43 \mathrm{~cm}$ y 5 ramas primarias. Sin embargo, tienen 2,77 cápsulas por nudo, peso de 1000 semillas 3,29 g y rendimiento de semilla 1415,35 kg ha-1.

Grupo 2: Accesiones de ajonjolí de porte grande con tallo púrpura verdoso, hojas lanceoladas o lineales

Conformado por 16 accesiones, presentan los dos tipos de crecimiento (determinado e indeterminado), el color del tallo es púrpura verdoso con diferentes tipos de pubescencia, ramificación opuesta, hojas lanceoladas o lineales, 
color del nectario extrafloral externo amarillo, color interno de la corola blanco con manchas rosadas, son mono 0 multicapsular y el color de la semilla se observa desde amarillo, café amarilloso con machas o rojizo hasta negro. Tienen los mayores promedios de altura con $158,6 \mathrm{~cm}$, longitud internudos de $87,3 \mathrm{~cm}, 6$ ramas primarias y 9 ramas secundarias. Así mismo, evidencia el mayor número de cápsulas por planta 295,29 y 81,58 cápsulas por semilla; peso de 1000 semillas $3,31 \mathrm{~g}$ y rendimiento de semilla $1325,15 \mathrm{~kg} \mathrm{ha}^{-1}$.

Grupo 3: Accesiones de ajonjoli de porte mediano con tallo amarillo verdoso, ramificación opuesta y semillas café

Conformado por 37 accesiones, las cuales tienen los dos tipos de crecimiento (determinado e indeterminado), hábito erguido, color del tallo amarillo, verde y ocasionalmente purpura verdoso, tipo de ramificación opuesta, hojas lanceoladas o lineales, posición de la hoja alterna y opuesta, color de nectario extrafloral externo amarillo claro, amarillo y amarillo verdoso, color interno de la corola blanco con manchas rosadas, son multicapsulares y el color de la semilla se aprecia desde café amarilloso hasta café. Muestran las hojas más anchas con 14,13 cm en la hoja basal y $8,96 \mathrm{~cm}$ en la hoja media. Sin embargo, registra el menor promedio con 124,86 cápsulas por planta y 73,94 semillas / cápsula, peso de 1000 semillas 3,39 g y rendimiento de semilla 1197,57 kg ha-1.

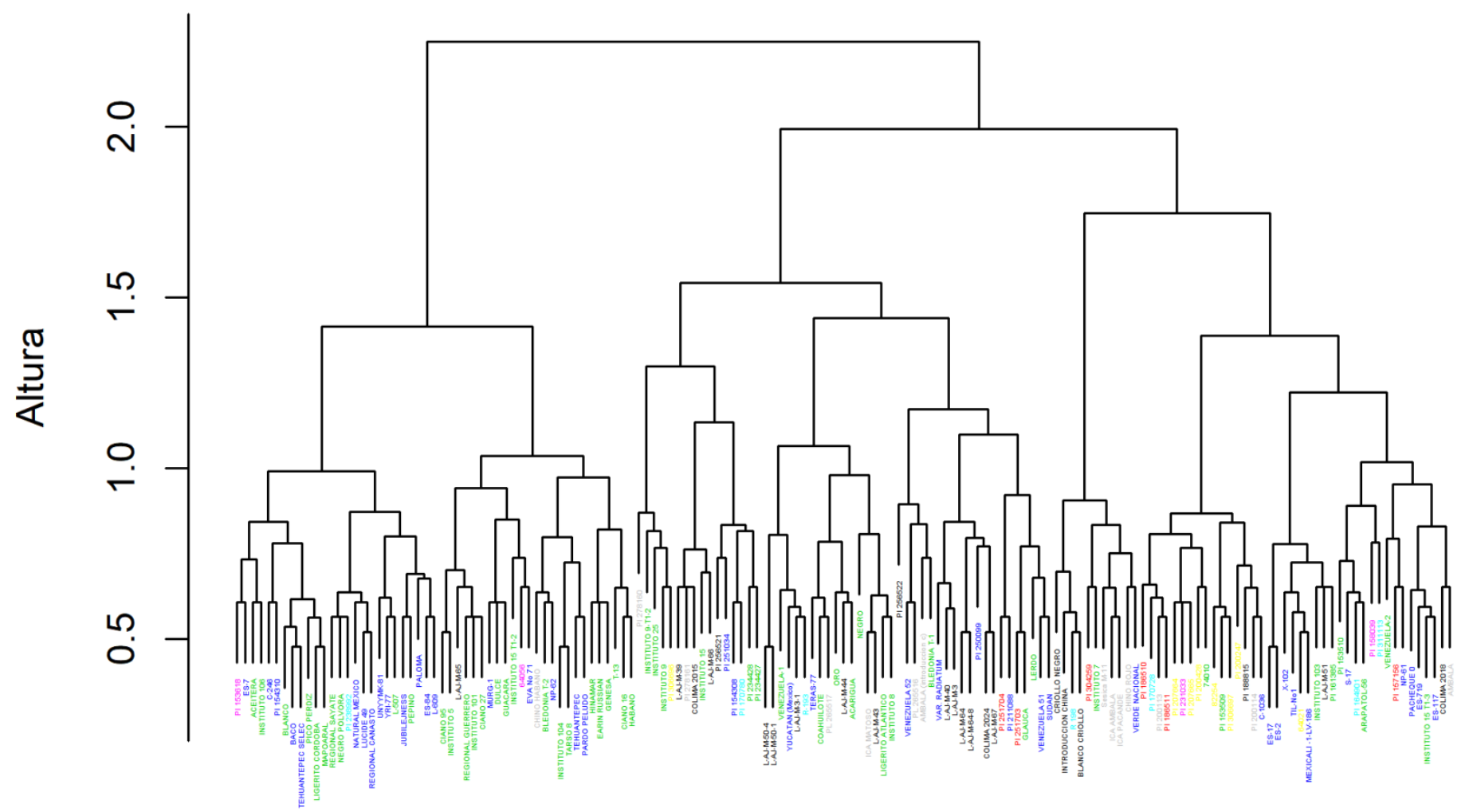

Figura 3. Dendrograma obtenido a partir de 25 variables discriminantes en las accesiones del banco de germoplasma de ajonjoli en Colombia. El color indica el origen de la accesión. Verde: Venezuela, Azul oscuro: México, Negro: sin información, Gris: Colombia, Azul aguamarina, Rojo, Amarillo y Fuscia: otros orígenes.

Grupo 4: Accesiones de ajonjolí de porte grande con tallo verde, ramificación decusada, hojas ovadas y semillas pálidas

Conformado por 11 accesiones con crecimiento determinado, hábito erguido, color del tallo verde, tipo de ramificación decusada, hojas ovadas, posición de la hoja decusada, color de nectario extrafloral externo amarillo claro y amarillo, desarrollo del nectario extrafloral rudimentario y pequeño, color interno de la corola blanco con manchas rosadas, son mono y multicapsulares y el color de la semilla se observa desde amarillo pálido hasta café pálido. Presentan los mayores promedios de altura con $158,52 \mathrm{~cm}, 15$ ramas primarias y 17 ramas secundarias. 
Tienen el menor número de semillas por cápsula 66,53 y por tanto el menor rendimiento de semilla con 1110,40 kg ha-1; sin embargo, el peso de 1000 semillas es $3,61 \mathrm{~g}$ siendo el mayor de los grupos.

Grupo 5: Accesiones de ajonjolí de porte pequeño con crecimiento determinado, tallo amarillo o verde y con manchas rosadas profundas en el interior de la corola

Conformado por 40 accesiones, se caracterizan por mostrar crecimiento determinado, hábito erguido, color del tallo amarillo, verde y amarillo verdoso, tipo de pubescencia glabra o débil, tipo de ramificación opuesta y alterna, posición de la hoja alterna, color de nectario extrafloral externo amarillo y raramente púrpura, color interno de la corola blanco con manchas rosadas profundas o rosado, son mono o multicapsulares y el color de la semilla se bserva desde amarillo pálido, café y ocasionalmente negro. Presentan los menores promedios de altura con 126,86 $\mathrm{cm}$ y 5 ramas primarias, así como, 2,28 cápsulas por nudo. El peso de 1000 semillas es $3,25 \mathrm{~g}$ y rendimiento de semilla más alto con $1789,75 \mathrm{~kg} \mathrm{ha}^{-1}$.

\section{B. Selección de accesiones representativas}

Se obtuvieron las distancias entre accesiones y el centro de su grupo, para determinar las más cercanas al centroide, es decir, las más representativas (Husson et al., 2010). En planta y plántula, el grupo uno, ORO y LUCIDE 49; grupo dos, TEHUANTEPEC SELEC y UNYYMK-81; grupo tres, L-AJ-M-51 y L-AJ-M-67; grupo cuatro, PI-306696 y PI-16385 y en el grupo cinco, PI-256522 y Pl-156618. En tallo y raíz, en el grupo uno, PARDO PELUDO y HABANO; grupo dos, PI-207664 y PI-232033 y en el grupo tres, INTRODUCCIÓN CHINA y CHINO ROJO.

En hoja, en el grupo uno, TEHUANTEPEC SELEC y NATURAL MÉXICO; grupo dos, ICA PACANDE y BLANCO CRIOLLO y en el grupo tres YUCATÁN y PI 265517. En flor, en el grupo uno, BACO y ES-719; grupo dos, PI311113 y BLEDONIA T-2 y en el grupo tres, EARIN RUSSIAN y CIANO 27. Finalmente, en cápsula y semilla, en el grupo uno, PI-157156 y L-AJ-M-3; grupo dos, ACEITERA y PI 265516 y en el grupo tres INSTITUTO 9-T1-2 e INSTITUTO 9.

Conocer la variación genética presente en el banco de germoplasma de ajonjolí en Colombia permite comprender la diversidad existente, crear estrategias de conservación e identificar genotipos promisorios para un programa de fitomejoramiento. La caracterización morfológica con 74 descriptores permitió identificar los caracteres discriminantes de las estructuras de la planta y la selección de variables morfológicas y de componentes de rendimiento para la identificación de conglomerados de manera conjunta. Estos estudios concuerdan con Laurentin (2017), al considerar en la obtención de nuevos genotipos los caracteres morfoagronómicos y de rendimiento.

El grupo planta y plántula fue discriminado por hábito, tipo de crecimiento, altura de planta, inserción y pilosidad del cotiledón. De acuerdo con Mustafa et al. (2015), la altura de la planta, número de ramas por planta, número de cápsulas por planta, semillas por cápsula y el peso de 1000 semillas son las características más asociadas a rendimiento. Lo que evidencia la importancia de estas variables en programas de mejoramiento de la especie; de igual forma, Falusi et al. (2015), identificaron la altura de la planta como una característica importante para diferenciar genotipos. En el grupo tallo y raíz se presentaron diferencias en la forma de la pubescencia, altura de la planta y número de ramas primarias. Cristaldo (2007) señala que algunas accesiones se caracterizan por ser glabras o tener pilosidad rala o escasa, y Falusi et al. (2015) identificaron el número de ramas por planta como determinante de la capacidad de carga.

En flor, la coloración de la corola, nectario extrafloral y número de flores por axila fueron discriminantes en la agrupación. Las diferentes formas de desarrollo del nectario extrafloral (rudimentario, pequeño, mediano y largo) concuerdan con los resultados obtenidos por Mazzani et al. (2007), quienes documentaron la mayor variabilidad para el carácter número de flores por axila y desarrollo de nectarios extraflorales. En relación con longitud de la 
corola, se presentó una media de 2,64 cm, resultados que son afines con los reportados por Azeez et al. (2017), quienes caracterizaron genotipos en un rango entre 2,03 - 3,27 cm.

La agrupación cápsula y semilla coincide con los dendrogramas generados por Bedigian et al. (1986), quienes separaron las accesiones por el número de carpelos de las cápsulas, plantas tetracarpeladas y bicarpeladas, contrario a lo encontrado por Mazzani et al. (2007), quienes no utilizaron la variable en el análisis de clasificación. En el presente estudio, la variable número de carpelos sirvió para la diferenciación de los grupos, en especial el grupo tres, al presentar 2,73 $\pm 0,18$ carpelos por encima de la media global 1,08 $\pm 0,29$. Según Delgado (1994), el número de cápsulas por planta es una característica que puede ser modificada por las condiciones ambientales en el momento de la floración y formación de frutos. La falta de agua o temperaturas elevadas ocasionan aborto de flores y cápsulas.

El número de cápsulas por nudo presentó una media global de 2,5 con valor mínimo de 1 y máximo 5.6, lo cual es consecuente con lo encontrado por Morris (2009), quien reporta entre una y cinco cápsulas por axila. En el número de semillas por cápsula se encontró un rango entre 60 - 85, valor que coincide con lo reportado por Zárate et al. (2011), al manejar un ciclo de siembra con distancia de 1 metro entre surcos y 6 plantas por metro lineal $(60.000$ plantas ha-1) y es superior a lo registrado por Laurentin et al. (2004) en la evaluación de ocho accesiones de ajonjolí de Venezuela.

Las accesiones ACEITERA, INSTITUTO 9-T1-2, PI-157156, PI 265516 e INSTITUTO 9 identificadas como promisorias en programas de mejoramiento, presentaron promedios de 222,7 cápsulas por planta, 92,92 semillas por cápsula y $3 \mathrm{~g}$ en peso de 1000 semillas, valores superiores a los hallados por Pérez-Bolaños y SalcedoMendoza (2018), quienes reportan estas variables como parte de los componentes de rendimiento en ajonjolí, y por Ismaila y Usman (2014) al considerar estas variables como elementos primarios en la producción de semilla.

\section{Conclusiones}

La agrupación de variables por estructura de la planta permite determinar los caracteres discriminantes facilitando la obtención de resultados en caracterizaciones morfológicas. A partir de esto, el proceso de mejoramiento genético en ajonjolí puede ser dirigido a una estructura específica. Las accesiones identificadas por cada estructura son potenciales parentales para la obtención de variedades promisorias que respondan a las demandas de la industria y mercado del ajonjolí. Las accesiones TEHUANTEPEC SELEC, L-AJ-M-67, L-AJ-M-51, PARDO PELUDO, BLANCO CRIOLLO, NP-61, BACO, INSTITUTO 104, CHINO ROJO, R 198 y ACARIGUA presentaron características de interés que diferencian los grupos formados. Las variables discriminantes y los materiales identificados permiten la generación de variedades para futuros programas de mejoramiento en Colombia.

\section{Agradecimientos}

Al Sistema de Bancos de Germoplasma de la Nación para la Alimentación y la Agricultura (SBGNAA) como fuente del material biológico. Esta investigación se desarrolló en el marco del convenio CN-44 DE 2012, suscrito entre el Instituto Colombiano Agropecuario (ICA) y la Corporación Colombiana de Investigación Agropecuaria (Corpoica) para la conservación y caracterización de los Bancos de Germoplasma de la Nación Colombiana. 


\section{Referencias}

Azeez, M. A., Olowookere, M. B., Animasaun, D. A., \& Bello, B. O. (2017). Utility of Some Floral Characters in the Assessment of Genetic Diversity in Sesame (Sesamum indicum L.). Acta Agriculturae Slovenica, 109(1), 61-70. https://doi.org/10.14720/aas.2017.109.1.06

Bedigian, D., Smyth, C. A., \& Harlan, J. R. (1986). Patterns of Morphological Variation in Sesamum indicum. Economic Botany, 40, 353-365. https://doi.org/10.1007/BF02858991

Cristaldo, R. (2007). Introducción y selección de cultivares de sésamo. En Jornada técnica científica del cultivo de sésamo (pp. 2-8). San Lorenzo, Campus Universitario.

Delgado, C. M. (1994). Correlations and Path Coefficients in Sesame (Sesamum indicum L.). Bioagro, 6(1), 18-23. https://www.researchgate.net/publication/295715460

Falusi, O. A., Yahaya, S. A., Gado, A. A., Daudu, O. A. Y., Akinbo, O. A., \& Teixeira da Silva, O. A. (2015). Morphological Evaluation of Selected Sesame (Sesamum indicum L.) Genotypes from Five States in Northern Nigeria. African Journal of Agricultural Research, 10(37), 3657-3661. https://doi.org/10.5897/AJAR2014.8928

Furat, S., \& Uzun, B. (2010). The Use of Agro-Morphological Characters for the Assessment of Genetic Diversity in Sesame (Sesamum indicum L.). Plant Omics, 3(3), 85-91. En https://www.researchgate.net/publication/228476285

Hata, N., Hayashi, Y., Okazawa, A., Ono, E., Satake, H., \& Kobayashi, A. (2012). Effect of Photoperiod on Growth of the Plants, and Sesamin Content and CYP81Q1 Gene Expression in the Leaves of Sesame (Sesamum indicum L.). Environmental and Experimental Botany, 75, 212-219. https://doi.org/10.1016/j.envexpbot.2011.07.004

Hidalgo, R. (2003). Variabilidad genética y caracterización de especies vegetales. En T. L. Franco \& R. Hidalgo (eds.), Análisis estadístico de datos de caracterización morfológica de recursos fitogenéticos (pp. 77-84). Boletín Técnico n. ${ }^{\circ} 8$, Instituto Internacional de Recursos Fitogenéticos IPGRI.

Husson, F., Lê, S., \& Pagès, J. (2010). Exploratory Multivariate Analysis by Example Using R. In Exploratory Multivariate Analysis by Example Using R. CRC Press. https://doi.org/10.1201/b10345

IPGRI \& NBPGR. (2004). Descriptors for Sesame (Sesamum spp.). International Plant Genetic Resources Institute, Rome, Italy; and National Bureau of Plant Genetic Resources, New Delhi, India.

Ismaila, A., \& Usman, A. (2014). Genetic Variability for Yield and Yield Components in Sesame (Sesamum indicum L.). International Journal of Science and Research (IJSR), 3(9), 63-66. http://dx.doi.org/10.5958/0975928X.2018.00189.8

Laurentin, H. (2017). UCLA-2, un nuevo cultivar de ajonjolí (Sesamum indicum L.) para los llanos occidentales de Venezuela. Bioagro, 29(1), 61-64. http://ve.scielo.org/scielo.php?script=sci_arttext\&pid=S1316-33612017000100007

Laurentin, H., Montilla, D., \& García, V. (2004). Relación entre el rendimiento de ocho genotipos de ajonjolí (Sesamum indicum L.) y sus componentes. Comparación de metodologías. Bioagro, 16(3), 153-162. http://ve.scielo.org/scielo.php?script=sci_arttext\&pid=S1316-33612004000300001 
Mazzani, E., Marín, C., \& Romero, J. R. (2007). Clasificación del banco de germoplasma de ajonjolí de Venezuela basada en escalas cualitativas. Agronomía Tropical, 57(1), 15-24. http://ve.scielo.org/scielo.php?script=sci_arttext\&pid=S0002-192X2007000100003

Ministerio de Tecnologías de la Información y las Comunicaciones -MINTIC-. (2018). Evaluaciones agropecuarias municipales EVA. https://www.datos.gov.co/Agricultura-y-Desarrollo-Rural/Evaluaciones-Agropecuarias-MunicipalesEVA/2pnw-mmge/data

Mooi, E., \& Sarstedt, M. (2011). Cluster Analysis. In E. Mooi \& M. Sarstedt (eds.), A Concise Guide to Market Research (pp. 237-284). Springer. https://doi.org/10.1007/978-3-642-12541-6

Morris, J. B. (2009). Characterization of Sesame (Sesamum indicum L.) Germplasm Regenerated in Georgia, USA. Genetic Resources and Crop Evolution, 56(7), 925-936. https://doi.org/10.1007/s10722-009-9411-9

Mustafa, H., Hasan, E., Ali, Q., Anwar, M., Aftab, M., \& Mahmood, T. (2015). Selection Criteria for Improvement in Sesame (Sesamum indicum L.). American Journal of Experimental Agriculture, 9(4), 1-13. https://doi.org/10.9734/AJEA/2015/17524

Pacheco, C. A., Vergara, M. C., \& Ligarreto, G. A. (2009). Clasificación de 85 accesiones de arveja (Pisum sativum L.), de acuerdo con su comportamiento agronómico y caracteres morfológicos. Agronomía Colombiana, 27(3), 323332. https://www.redalyc.org/articulo.oa?id=180316242005

Pagès, J. (2004). Analyse factorielle de donnees mixtes: príncipe et exemple d'application. In Actes des XXXVlémes Journées de Statistique á Montpellier. http://www.agro-montpellier.fr/sfds/CD/textes.htm

Pérez-Bolaños, J. D. J., \& Salcedo-Mendoza, J. G. (2018). Componentes del rendimiento en cultivares de ajonjolí Sesamum indicum L. (Pedaliaceae), en el departamento de Sucre (Colombia). Ciencia y Tecnología Agropecuaria, 19(2), 263-273. https://doi.org/10.21930/rcta.vol19_num2_art:660

Pham, T. D., Nguyen, T. D. T., Carlsson, A. S., \& Bui, T. M. (2010). Morphological Evaluation of Sesame (Sesamum indicum L.) Varieties from Different Origins. Australian Journal of Crop Science, 4(7), 498-450. https://www.researchgate.net/publication/235984063

R Core Team (2017). A Language and Environment for Statistical Computing. R Foundation for Statistical Computing. https://www.r-project.org/

Rey, L. (1999). Recursos genéticos y mejoramiento. En L. Rey, E. Barragán, P. P. Herrera, E. Echeverry, A. Henao, M. D. Lozano, L. E. Gómez, R. Simmods, H. L. Vargas, \& N. Sánchez (eds.), El cultivo de ajonjolí, producción y utilización (p. 200). Corpoica, Regional 6.

Valencia, R. A., Lobo A, M., \& Ligarreto M, G. A. (2010). Estado del arte de los recursos genéticos vegetales en Colombia: sistema de bancos de germoplasma. Corpoica Ciencia y Tecnología Agropecuaria, 11(1), 85-94. https://doi.org/10.21930/rcta.vol11_num1_art:198

Zárate, C. L., Oviedo, R. M., \& González, D. D. (2011). Rendimiento del cultivo de sésamo (Sesamum indicum L.), variedad Mbarete, en diferentes épocas de siembra y poblaciones de plantas. Investigación Agraria, 13(2), 67-74. https://www.researchgate.net/publication/319335760 
Cómo citar este artículo: Garnica, J., Rodríguez, O., Jaramillo-Barrios, C., Villamil, J. \& Valencia, J. (2020). Caracterización morfológica de 160 accesiones de ajonjolí (Sesamum indicum L.) del banco de germoplasma de Colombia. Cien. Agri. $17(3), 63-77$. DOI: https://doi.org/10.19053/01228420.v17.n3.2020.11618 